\title{
Magnetism of ferriprotoporphyrin IX monomers and dimers
}

\author{
J. Stanek*, K. Dziedzic-Kocurek \\ Marian Smoluchowski Institute of Physics, Jagiellonian University, 30-059 Kraków, Reymonta 4, Poland
}

\section{A R T I C L E I N F O}

\section{Article history:}

Received 27 December 2008

Received in revised form

6 November 2009

Available online 16 December 2009

Keywords:

Protoporphyrin IX

Oxo-dimer

Magnetic relaxation

Mössbauer spectroscopy

SQUID

\begin{abstract}
A B S T R A C T
The SQUID and the ${ }^{57} \mathrm{Fe}$ Mössbauer spectroscopy studies of the magnetic properties of monomeric and dimeric forms of iron porphyrin were performed between 2 and $305 \mathrm{~K}$. The effective magnetic relaxation rate of the Fe atoms in iron porphyrin monomers exhibits complex temperature dependence, resulting from the competing spin-spin and spin-lattice relaxation processes. The dimerization of iron porphyrin dramatically speeds up the magnetic relaxation. The $\mathrm{Fe}-\mathrm{Fe}$ antiferromagnetic exchange coupling constant in Fe-O-Fe dimer is $J \approx-110 \mathrm{~cm}^{-1}$. The complementary application of SQUID and the Mössbauer spectroscopy is proposed as a new precise quantitative analytical methodology for monitoring of the aggregation process of iron porphyrin.
\end{abstract}

(c) 2009 Elsevier B.V. All rights reserved.

\section{Introduction}

Porphyrins and metalloporphyrins take part and play a crucial role in many important biochemical processes, especially those that are related to electron transfer. Porphyrin derivatives also attract attention because of potential clinical applications [1], for example, as photosensitizing agents in the photochemotherapy of cancer [2-4]. Metalloporphyrins, because of their electrical, optical, and magnetic properties, are promising candidates for photoconducting and charge storing materials [5]. The dimerization and oligomerization of iron porphyrins have been studied in various model systems for more than 30 years. However, the mechanism of interaction between the heme centers remains obscure. The most often investigated systems of dimerized iron porphyrins are $\mu$-oxo $\mathrm{Fe}-\mathrm{O}-\mathrm{Fe}$ or peroxo bridged $\mathrm{Fe}-\mathrm{O}-\mathrm{O}-\mathrm{Fe}$ complexes. The scheme of the structure of the iron porphyrin molecule and one of its possible dimers are shown in Fig. 1. Due to the complicated preparative work and the low symmetry of the molecule, derivatives of the porphyrin IX are relatively rarely studied, although this porphyrin plays the most important role in life processes.

The influence of the aggregation of porphyrin on the local magnetic and dynamical properties of iron has been studied by us previously [6] using EXAFS (extended X-ray absorption fine structure) and Mössbauer spectroscopy techniques. Comparison of the results obtained by these two methods enabled separation

\footnotetext{
* Corresponding author. Tel.: +48126635537.

E-mail address: Jan.Stanek@uj.edu.pl (J. Stanek).
}

of the individual Fe ion vibration from its collective movement with ligands. However, reported preliminary data on the temperature dependence of the magnetic relaxation times in these systems could not be satisfactorily explained. In this paper, we focus only on magnetic properties of the iron porphyrin monomers and dimers. Application of the SQUID and the Mössbauer spectroscopy joins fruitfully the macro- and microscopic insight, respectively.

\section{Experimental}

\subsection{Samples}

The iron porphyrin dimers, enriched in a stable ${ }^{57} \mathrm{Fe}$ isotope (Mössbauer probe), were prepared by insertion of the iron atoms from ${ }^{57} \mathrm{FeCl}_{3}$ into protoporphyrin IX (Sigma) and finally precipitated from water- $N, N$-dimethylformamide (DMF) solution, according to the modified Adler procedure [7]. The obtained specimen and the commercial ferriprotoporphyrin IX chloride (Fe-PPIX-Cl) (Alfa Aesar) were tested by UV/vis absorption and fluorescence spectroscopy, by FTIR (Fourier transformed infrared spectroscopy) and Raman spectroscopy. The analysis confirmed formation of the $\mu$-oxo-bridges between Fe atoms, i.e. dimerization of the ferriprotoporphyrin IX rings. Next, the same samples synthesized by the same preparation run were examined by Mössbauer spectroscopy and EXAFS technique between 5 and $305 \mathrm{~K}$. Observed significant difference of the local dynamical iron properties between the porphyrin monomers and the prepared specimen was an additional microscopic indication of the dimerization of ferriprotoporphyrin IX [6]. 

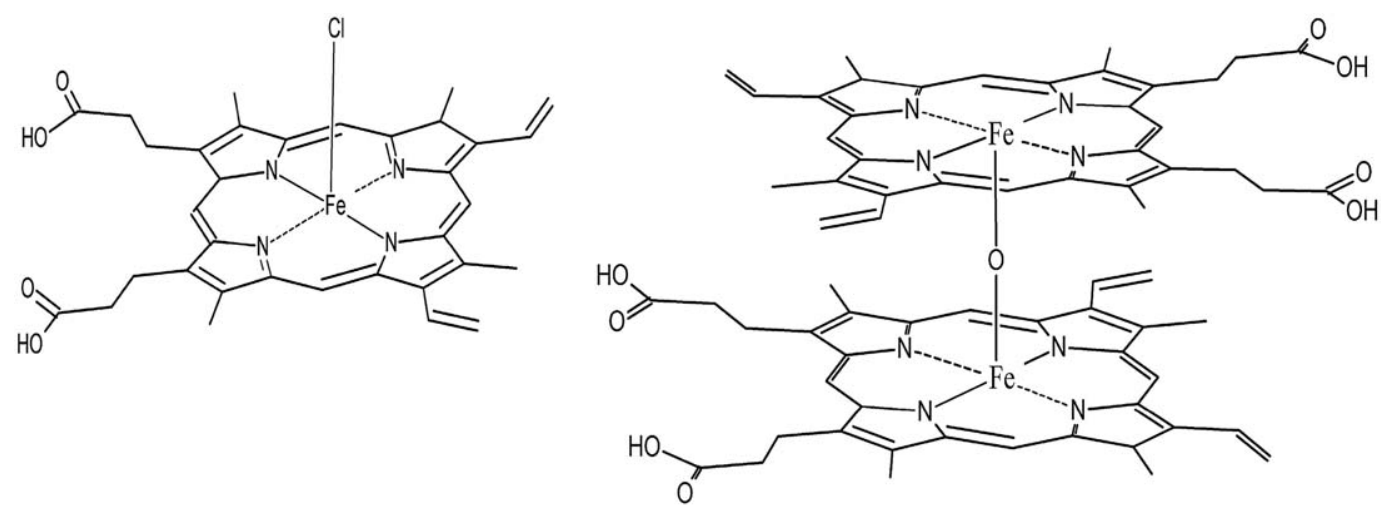

Fig. 1. Molecular structure of the iron porphyrin monomer, left, and iron porphyrin dimer, right.

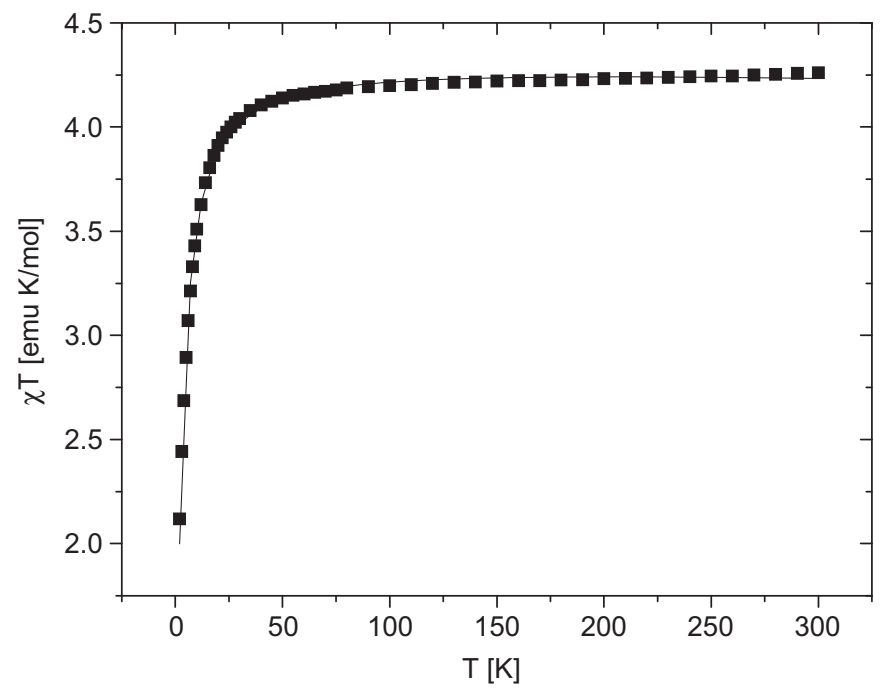

Fig. 2. Temperature dependence of the magnetic susceptibility, $\chi$, multiplied by temperature, $T$, of iron porphyrin monomers. The solid line is the fit with $g=1.99$ and $S=5 / 2,(H=1000$ Oe $)$.

\subsection{Susceptibility measurements}

The susceptibility, $\chi$, of the ferriprotoporphyrin IX chloride (FePPIX-Cl) monomers and of the sample containing iron porphyrin dimers was measured by the SQUID method, between 2 and $300 \mathrm{~K}$. The magnetic field applied was 1000 Oe. Although such rather strong field may disturb the proportionality between magnetization and susceptibility at very low temperatures, such fields (or stronger) were applied in the past by other authors [8] for similar systems, which enables the comparison of the obtained results. The mass of the specimen was $7.3 \pm 0.1$ and $2.5 \pm 0.1 \mathrm{mg}$ for Fe-PPIX-Cl and dimers containing sample, respectively. The dependence of $\chi T$ versus $T$ for measured samples is shown in Figs. 2 and 3.

\subsection{Mössbauer spectroscopy study}

The ${ }^{57} \mathrm{Fe}$ Mössbauer spectra were recorded in the transmission mode between 25 and $306 \mathrm{~K}$, in the Leyboldt gas-flow cryostat. The thickness of the powder sample (Fe-PPIX-Cl) with a natural abundance of Fe has been optimized to $203 \mathrm{mg} / \mathrm{cm}^{2}$, according to the Long procedure [9], while the sample containing dimers, enriched in ${ }^{57} \mathrm{Fe}$ up to $97 \%$, contained $5 \mathrm{mg} / \mathrm{cm}^{2}$ specimen mixed with $100 \mathrm{mg}$ lucid powder. Selected spectra are shown in Figs. 4 and 5.

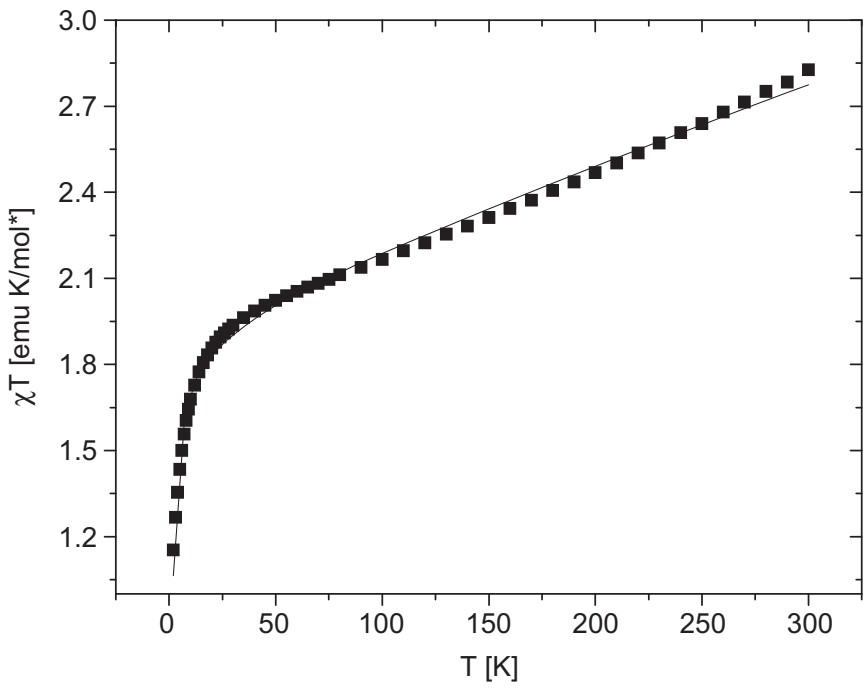

Fig. 3. Temperature dependence of the magnetic susceptibility, $\chi$, multiplied by temperature, $T$, of sample containing iron porphyrin dimers. The solid line is the fit, assuming contribution from the two fractions - monomers and dimers ( $H=1000 \mathrm{Oe}),[\mathrm{mol} *]$ - weighted-average molar mass.

\section{Results}

\subsection{SQUID measurements}

Measured magnetic susceptibility, $\chi$ (Eq. (1)) in the case of monomer iron porphyrin sample, contains the signal from paramagnetic Fe ions with spin $S=5 / 2, \chi_{\text {mon, which originates }}$ from the intermolecular interactions, and the diamagnetic contribution of remaining atoms, $\chi_{\text {dia }}$ :

$\chi=\chi_{\text {mon }}+\chi_{\text {dia }}$

The description and the interpretation of the temperature dependence of the susceptibility may be done within two competing models. The first one, based on the crystal field theory, has been applied by physicists for iron porphyrins already in the 1960 s of the previous century $[10,11]$. However, it seems that this approach, which assumes predominantly ionic interactions of the metal atoms with ligands, being very useful in inorganic coordination chemistry, has limited application to the description of the properties of porphyrins. In fact "there is a great deal of evidence that in no state of any iron-porphyrin heme protein are the electromagnetic properties of the iron close to those in its free-ion state" [12]. Thus, we apply the model that neglects the crystal field splitting of the $3 \mathrm{~d}^{5}$ state of the high spin $\mathrm{Fe}^{3+}$ but includes the weak intermolecular magnetic exchange interaction, in the 


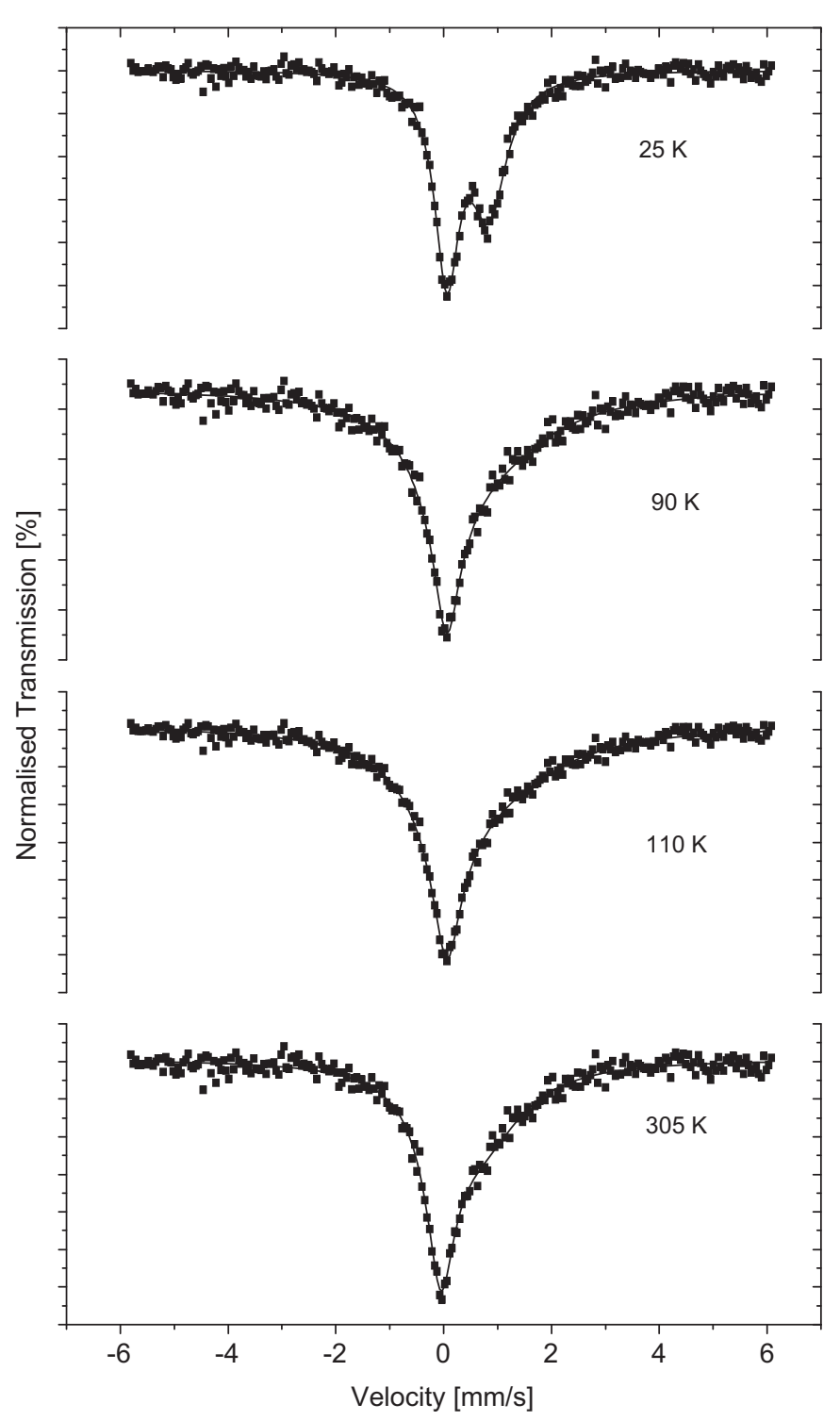

Fig. 4. Selected ${ }^{57} \mathrm{Fe}$ Mössabauer spectra of iron porphyrin at temperatures as shown.

form of $z J\left\langle S_{z}\right\rangle S_{z}$. Here, $\left\langle S_{z}\right\rangle$ is the mean value of the $S_{z}$ component of the spin operator of the $z$ nearest neighboring atoms around a given magnetic molecule in the crystal lattice and $J$ is the exchange integral [13].These weak magnetic molecular interactions modify the Curie law, Eq. (2), to the form expressed by Eq. (3) [13]:

$\chi=\frac{N g^{2} \mu_{\mathrm{B}}^{2} S(S+1)}{3 k T}$

$\chi_{m o n}=\frac{N g^{2} \mu_{\mathrm{B}}^{2} S(S+1)}{3 k T-z J S(S+1)}$

The diamagnetic contribution $\chi_{\text {dia }} \approx-250 \times 10^{-6} \mathrm{emu} / \mathrm{mol}$ was estimated from the Pascal constants [13]. The diamagnetic signal of the sample holder was subtracted based on an independent experiment. Its contribution to the total spectra increases in the higher temperature region. The experimental temperature dependence of $\chi$ multiplied by $T$ was fitted to Eq. (3). The fit with $g$-factor equal $g=1.99 \pm 0.1$ and $z J=-0.6 \pm 0.1 \mathrm{~cm}^{-1}$ reproduces the experimental data, cf. Fig. 2.

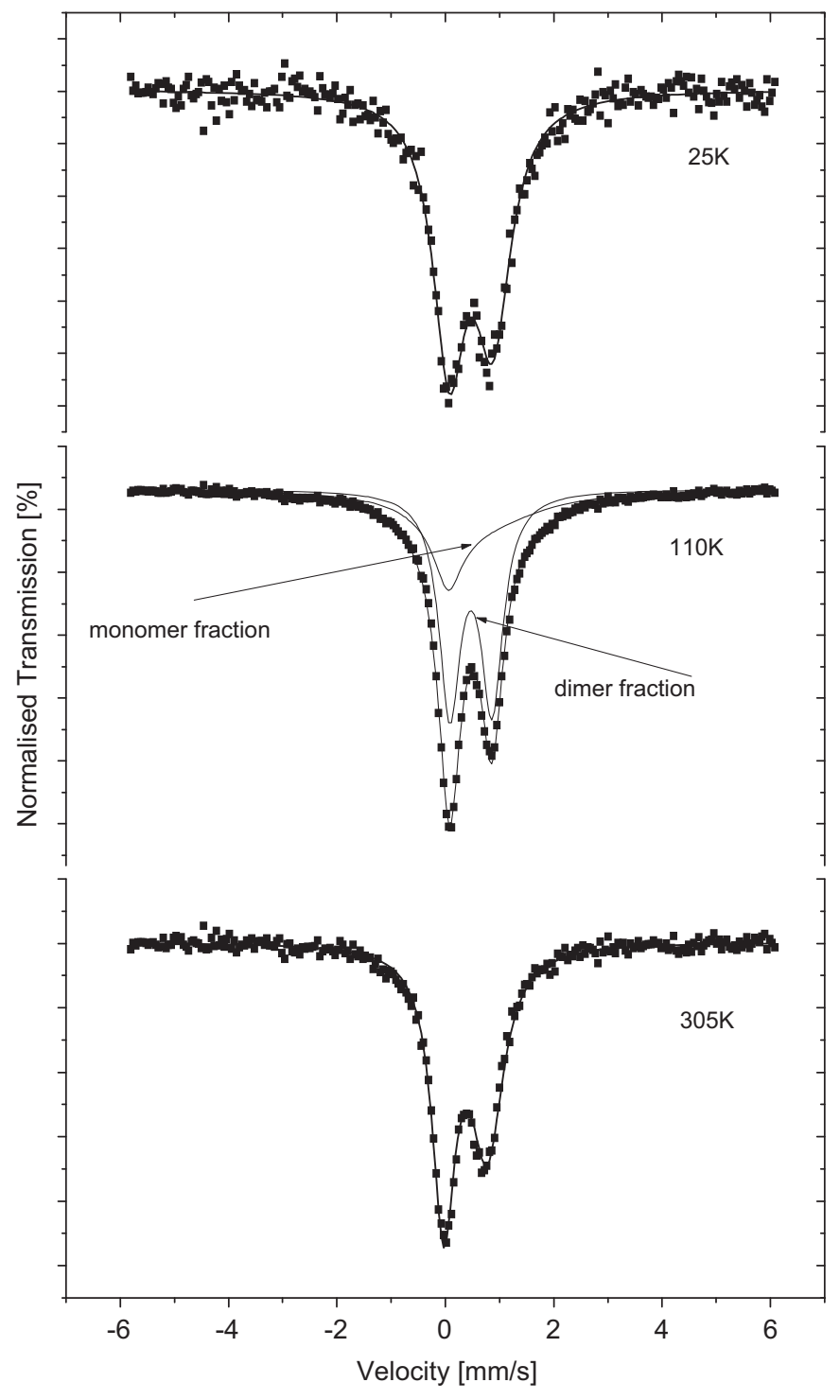

Fig. 5. Selected ${ }^{57} \mathrm{Fe}$ Mössabauer spectra of the sample containing porphyrin monomers and dimers at temperatures as shown. The solid lines are the fits assuming two fractions: relaxation spectrum of the monomers and symmetric doublet of dimers.

The temperature dependence of the susceptibility of the sample containing iron porphyrin dimers could not be reproduced by any simple model. Again, we ignore the crystal field splitting of $\mathrm{Fe}^{3+}$ ions, but now the spin-spin interaction within the dimer may not be treated as the perturbation to the Zeeman term, $\hat{H}_{\text {ZEE }}$. In general, the susceptibility of the sample containing $\mathrm{Fe}^{+3}$ iron dimers, $\chi_{\text {dim }}$, may be derived as follows. The magnetic interaction between two spins $S_{1}$ and $S_{2}$ is described by the HeisenbergDirac-Van Vleck spin Hamiltonian, $\hat{H}_{\mathrm{HDVv}}$. The total spin Hamiltonian for interpretation of magnetic measurements takes the following form:

$\hat{H}=\hat{H}_{\mathrm{ZEE}}+\hat{H}_{\mathrm{HDVV}}$

$\hat{H}=g \mu_{\mathrm{B}} \hat{S}_{z} H_{z}-2 J \hat{S}_{1} \hat{S}_{2}$

In the described case of the dimer $S_{1}=S_{2}=5 / 2$. The total spin of the dimer, $S_{\text {eff, }}$ may take values $0,1,2,3,4$, and 5 . The intramolecular magnetic interactions are represented by $\chi_{\text {dim, }}$ which arise from the Zeeman splitting of the corresponding energy levels and their Boltzman population may be expressed by 


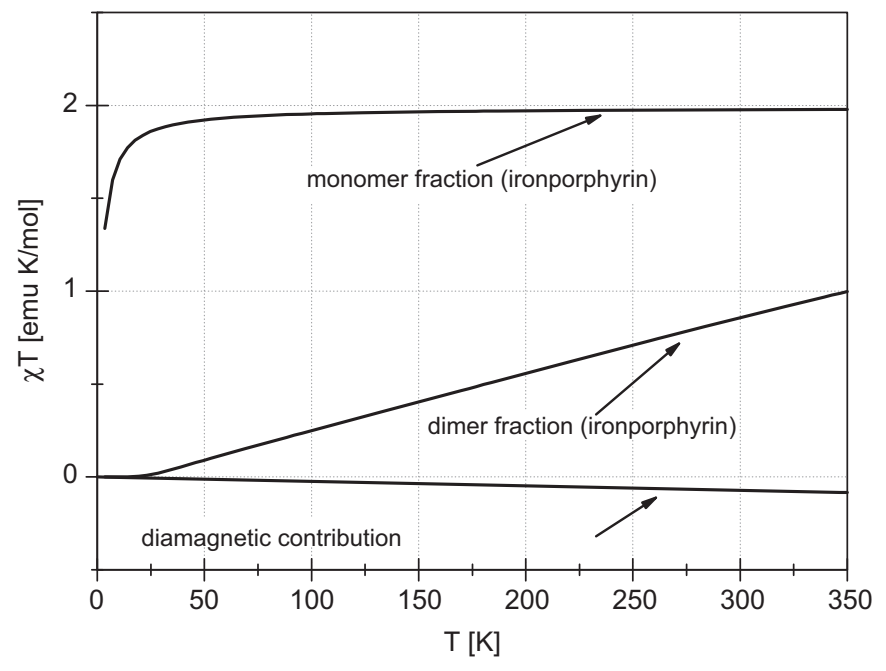

Fig. 6. Modeled magnetic susceptibility $T \chi(T)$ contribution of each of the magnetic fraction.

Eq. (6) [14]:

$\chi_{\operatorname{dim}}=\frac{N g^{2} \mu^{2}}{k T} \frac{e^{J / k T}+5 e^{3 J / k T}+14 e^{6 J / k T}+30 e^{10 J / k T}+55 e^{15 J / k T}}{1+3 e^{J / k T}+5 e^{3 J / k T}+7 e^{6 J / k T}+9 e^{10 J / k T}+11 e^{15 J / k T}}$

This apparently complicated expression describes the situation where two iron magnetic moments are coupled antifferromagnetically, forming a diamagnetic center with $S_{\text {eff }}=0$ at low temperatures. Resulting from Eq. (7) the $\chi_{\operatorname{dim}} T(T)$ dependence is shown in Fig. 6. It turns out that the $\chi_{\operatorname{dim}} T(T)$ susceptibility curve of the antiferromagnetically coupled dimers has a TIP-like (temperature independent paramagnetism) shape, which is hardly distinguishable from ferri-magnetic nano-particles (rust) [13]. The existence of such pollutions in the studied sample was excluded by Mössbauer experiments. Thus, SQUID measurement confirmed the formation of the antiferromagnetically coupled Fe dimers, but, in addition, it pointed out the presence of an admixture of iron porphyrin monomer in the specimen, which hardly could be identified from the applied analytical methods. Consequently, the susceptibility of this sample may be expressed by Eq. (7):

$\chi=(1-\rho) \chi_{\text {mon }}+\rho \chi_{\text {dim }}+\chi_{\text {dia }}$

where $\rho$ is the molar mass fraction. The fit of Eq. (7) with conditions given by Eqs. (6) and (3) to the experimental data, shown in Fig. 3, yields $\rho=0.59, J=-110 \pm 15 \mathrm{~cm}^{-1}$, and $g=2.1 \pm 0.2$. It has turned out that only $60 \%$ of the iron porphyrin is in the dimerized form. Contributions to each of the sample fractions to $\chi T(T)$, modeled with the parameters obtained from the fit, are shown in Fig. 6.

\subsection{Mössbauer measurements}

Description of the magnetic hyperfine structure of the Mössbauer spectra of the diluted paramagnetic $\mathrm{Fe}^{3+}$ ions in the organic materials belongs to the most challenging subjects in Mössbauer spectroscopy. In general, it requires application of the spin Hamiltonian formalism [15]. For $S=5 / 2$ and nuclear spins, $I_{\mathrm{e}}=3 / 2$ and $I_{\mathrm{g}}=1 / 2$, for the excited and ground states, respectively, it leads to the consideration $(2 S+1) \times\left(2 I_{\mathrm{e}}+1\right) \times(2 S+1) \times\left(2 I_{\mathrm{g}}+1\right)=288$ transitions in the Mössbauer spectrum. If the relaxation must be included, as in the studied case, any attempt of the exact fitting of the spectra is rather doubtful. Moreover, the adaptation of the crystal field theory to porphyrins is questionable, cf. Section 3.1, and

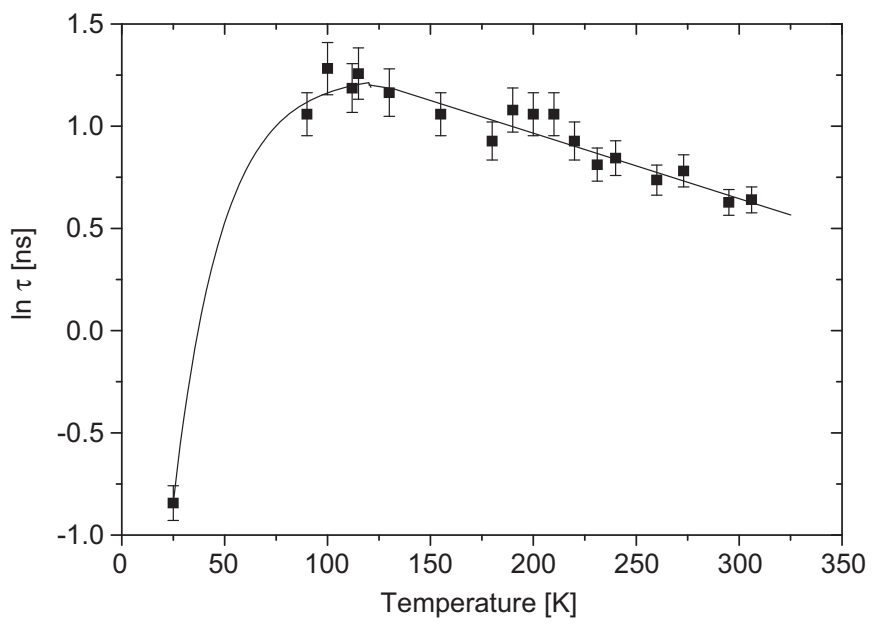

Fig. 7. The temperature dependence of the magnetic relaxation time $\tau$ of $\mathrm{Fe}^{3+}$ in iron porphyrin.

the crystal field parameters, obtained from the formal fit of the relaxation Mössbauer spectra have limited physical meaning. Thus, to parameterize the temperature evolution of the shape of the absorption lines, which is obvious from the visual inspection of the data, the Blume-Tjon model [16] was applied to fit the spectra, which were corrected for the finite absorber thickness [17]. The Blume-Tjon model parameterizes the hyperfine nuclear levels in terms of the effective hyperfine magnetic field that fluctuates only between parallel and antiparallel orientation versus the main axis of the axial electric field gradient. Additionally, we constrained average times, $\tau$, which the hyperfine field acts in the parallel and antiparallel directions as equal and assigned it to the effective relaxation time. In spite of these serious simplifications, the spectra could be well reproduced with only few fitting parameters: line width, quadrupole splitting, magnetic field, isomer shift, and relaxation time. The temperature dependence of $\tau$ of the iron porphyrin sample is shown in Fig. 7. Determined values of $\tau$ should be taken only as a phenomenological parameter. However, an increase in the relaxation rate, $1 / \tau$, at the low temperature, its minimum at about $100 \mathrm{~K}$, followed by a further increase at higher temperature, is obvious.

The spectra of the sample containing iron porphyrin dimers were fitted by two components: according to the SQUID results, $40 \%$ of the iron porphyrin synthesized sample was set as the monomer fraction. Differences in the recoil free fractions for both phases [6] were taken into account. Values of the hyperfine parameters of the iron porphyrin fraction at each temperature were fixed to these obtained in the single-phase monomeric iron porphyrin sample. It turned out that the remaining subspectrum of the dimers is a symmetric quadrupole doublet.

\section{Discussion}

One of the main problem in biophysical research of porphyrins is to separate the results specific for the peculiar, studied sample, from the general conclusions concerning the studied problem. At first sight, the dimerization of the iron porphyrin, obtained according to the broadly accepted procedure [7], only modifies some of the iron porphyrin properties [6], cf. for example, the shape of the Mössbauer spectra of porphyrin monomers and "dimers" in Figs. 4 and 5. The application of SQUID and Mössbauer spectroscopy leads to the somewhat frustrating, but clear and honest conclusion: applied chemical procedure transforms only partly the iron porphyrin monomers into dimers and the obtained 
specimen is a mixture of two phases. The complementary macroscopic (SQUID) and microscopic (Mössbauer) test of the magnetic properties may be applied in the preparatory biochemistry as an additional analytical procedure for unambiguous monitoring of the aggregation process of iron porphyrin.

The iron in iron porphyrin is in the high spin, $S=5 / 2, \mathrm{Fe}^{3+}$ state and determined susceptibility is a handbook example of the paramagnetic specimen, with weak antiferromagnetic intermolecular interactions. The magnitude of these interactions, $z J S(S+1) \approx-5 \mathrm{~cm}^{-1}=6 \times 10^{-4} \mathrm{eV}=7 \mathrm{~K}$, which is comparable with the crystal field parameter $D \approx 7 \mathrm{~cm}^{-1}$, determined for the similar systems $[10,11]$, hints that both models, namely crystal field approach and exchange interactions lead to the similar value of the energy needed for the reorientation of $5 / 2$ spin of $\mathrm{Fe}^{3+}$. However, the existence of the exchange interaction may be identified as the mechanism of the spin-spin relaxation, observed by Mössbauer spectroscopy in this rather diluted monomer system. In the crystal field theory, the probabilities of the transitions among combined ionic and nuclear states are phenomenological parameters.

The direction of the iron spin fluctuates rather slowly. The "effective" relaxation rate is in the range of $5 \times 10^{8} \mathrm{~s}^{-1}$, i.e. during the lifetime of the excited $14.4 \mathrm{keV}$ nuclear level of ${ }^{57} \mathrm{Fe}$, $\tau_{\mathrm{n}}=141 \mathrm{~ns}$, which is the observation time of the ${ }^{57} \mathrm{Fe}$ Mössbauer spectroscopy, the magnetic moment of the iron changes its direction about 50 times and reaches the Larmor precession time, $\tau_{\mathrm{L}}=\hbar / \mu_{\mathrm{n}} H \approx 5 \mathrm{~ns}$ for $H=500 \mathrm{kG} \mathrm{s}$, the typical value for the hyperfine field for $\mathrm{Fe}^{3+}$ ions. The $\mu_{\mathrm{n}}$ is magnetic moment of the nucleus, different for ground and excited states. The spin-spin relaxation depends on the concentration of magnetic ions being, essentially, independent of the temperature. The relaxation time is characteristic for the specific electronic level. Thus, at low temperatures, when only the lowest, fast relaxing electronic states are populated, the increase in the spin-spin relaxation rate may be observed as in the reported case of the iron porphyrin monomers. Such surprising effect was observed and explained in the past by Blume [10] who, in the frame of the crystal field theory, assigned these lowest electronic states to the Kramers doublet with $S_{z}= \pm 1 / 2$, where $z$ is the main axis of the electric field gradient tensor, here perpendicular to the pyrole ring plane. Accordingly, the Kramers doublets with $S_{z}= \pm 5 / 2, \pm 3 / 2$ are equally populated at higher temperature, which stabilizes the spin-spin relaxation rate. Similar example, however, describing more magnetically diluted, probably with lower $z J S(S+1)$ value and therefore slower relaxing $\mathrm{Fe}^{3+}$ system in metmyoglobin has been studied by Bizzari et al. [18].

The spherical charge symmetry of the ${ }^{6} \mathrm{~S}$ state of the high spin $\mathrm{Fe}^{3+}$ favors the spin-spin process of the relaxation, especially in the low temperature limit. At higher temperature, when the spinspin relaxation time stabilizes, the contribution of the spin-lattice relaxation becomes visible. The model of the spin-lattice relaxation was proposed by Van Vleck [19]. The spin-lattice relaxation rate increases with the temperature in a complicated way, depending on the temperature range, crystal field splitting and the Debay temperature. It is interesting to note that at low temperature, when only the states with $S_{z}= \pm 1 / 2$ are populated, the spin-lattice relaxation, in particular this related to the onephonon process, vanishes. Consequently, the temperature dependence of the total relaxation time may be quite complicated as in the reported case, see Fig. 7.

The dimerization of iron porphyrins leads to the formation of "diamagnetic" at $0 \mathrm{~K}$ system, which becomes weakly paramagnetic at elevated temperature due to the thermal excitation of the states with $S_{\text {eff }}=1,2,3$, and 5 . The determined from the magnetic susceptibility value of the exchange integral, $J \approx-110 \mathrm{~cm}^{-1}$, in the range reported for by different authors [14,20-22] for the Fe-Fe coupling via oxo-bridges. The interpretation of the Mössbauer spectra must be based on the local character of this method, which probes the hyperfine field at the ${ }^{57} \mathrm{Fe}$ nucleus, inside the magnetic atom, not in the diamagnetic molecule. The spectrum in the form of the symmetric quadrupole paramagnetic doublet with vanished Zeeman interactions proves that such dimer is a dynamical system and the rate of magnetic relaxation of the individual $\mathrm{Fe}^{+3}$ magnetic moments exceeds $10^{10} \mathrm{~s}^{-1}$. It seems reasonable to assume that the reorientation of the iron spins is correlated within the dimer via $\mu$-oxo bridge.

\section{Acknowledgements}

The authors thank Dr. Michał Rams and Michał Kwaśniak for carrying out the SQUID measurements and fruitful discussions. This work was supported by HASYLAB-DESY, Hamburg, Germany under contract RII3-CT-2004-506008-DESY-D-II-20060195 EC.

\section{References}

[1] T.J. Egan, Recent advances in understanding the mechanism of hemozin (malaria pigment) formation, J. Inorg. Biochem. 102 (2008) 1288-1299.

[2] R. Muschter, Photodynamic therapy: a new approach to prostate cancer, Curr. Urol. Rep. 4 (2003) 221-228.

[3] K. Berg, P.K. Selbo, A. Weyerang, A. Dietze, L. Prasmickaite, A. Bonsted, B.O. Engesaeter, E. Angell-Petersen, T. Warloe, N. Frandsen, A. Hogset, Porphyrinrelated photosentizers for cancer imaging and therapeutic applications, J. Microsc. 218 (2005) 133-147.

[4] K. Koenig, M.T. Wyss-Desserich, Y. Tadir, U. Haller, B. Tromberg, M.W. Berns, P. Wyss, Modifications of protoporphyrin IX fluorescence during photodynamic therapy of endometriosis, Med. Laser Appl. 21 (2006) 291-297.

[5] T. Yokoyama, S. Yokoyama, T. Kamikado, Y. Okuno, S. Mashiko, Selective assembly on surface of supramolecular aggregates with controlled size and shape, Nature 413 (2001) 619-621.

[6] K. Dziedzic-Kocurek, J. Stanek, K. Burda, Dynamics of iron in Fe-porphyrin aggregates studied by X-ray absorption and Mössbauer spectroscopy, Hyperfine Interact. 185 (2008) 87-97.

[7] A.D. Adler, F.R. Long, F. Kampas, J. Kim, On the preparation of metalloporphyrins, J. Inorg. Nucl. Chem. 32 (1970) 2443-2445.

[8] S. Mitra, Iron Porphyrins. Part II, in: A.B.P. Lever, H.B. Gray (Eds.), AddisonWesley Publishing Company, London, Amsterdam, Ontario, Sydney, Tokyo, 1983.

[9] G.J. Long, T.E. Cranshaw, G. Longworth, The ideal Mössbauer effect absorber thicknesses, Mössbauer Effect Ref. Data J. 6 (1983) 42-49.

[10] M. Blume, Temperature-dependent spin-spin relaxation times: application to the Mössbauer spectra of ferric hemin, Phys. Rev. Lett. 18 (1967) 305-308.

[11] G. Lang, T. Asakura, T. Yonetani, Magnetic hyperfine interactions in dilute hemin, Phys. Rev. Lett. 24 (1970) 981-983.

[12] G.H. Loew, Iron Porphyrins. Part II, in: A.B.P. Lever, H.B. Gray (Eds.), AddisonWesley Publishing Company, London, Amsterdam, Ontario, Sydney, Tokyo, 1983.

[13] O. Kahn, in: Molecular Magnetism, VCH Publishers, New York, 1993.

[14] K.S. Murray, Binuclear oxo-bridged iron(III) complexes coord, Chem. Rev. 12 (1974) 1-35.

[15] H.H. Wickman, M.P. Klein, D.A. Shirley, Paramagnetic hyperfine structure and relaxation effects in Mössbauer spectra: ${ }^{57} \mathrm{Fe}$ in ferrichrome, Phys. Rev. A 152 (1966) 345-357.

[16] M. Blume, J. Tjon, Mössbauer spectra in fluctuating environment, Phys. Rev. 165 (1968) 446-456.

[17] D.G. Rancourt, J.Y. Ping, Voigh-based methods for arbitrary-shape static hyperfine parameter distributions in Mössbauer spectroscopy, Nucl. Instrum. Methods Phys. Res. B58 (1991) 85-97.

[18] A.R. Bizzari, O.A. Iakoleva, F. Parak, Spin-lattice relaxation in Mössbauer spectra of metmyoglobin: investigatioon of crystals, water and waterglycerol solutions, Chem. Phys. 191 (1995) 185-194.

[19] J.H. Van Vleck, Paramagnetic relaxation times for titanium and chrome alum, Phys. Rev. 57 (1940) 426

[20] G.N. La Mar, G.R. Eaton, R.H. Holm, F.A. Walker, Proton magnetic resonance investigation of antiferromagnetic oxo-bridged ferric dimers and related high-spin monomeric ferric complexes, J. Am. Chem. Soc. 95 (1973) 63-75.

[21] D.M. Kurtz, Oxo- and hydroxo-bridged diiron complexes: a chemical. perspective on a biological unit, Chem. Rev. 90 (1990) 585-606.

[22] S. Mitra, Iron Porphyrins. Part II, in: A.B.P. Lever, H.B. Gray (Eds.), AddisonWesley Publishing Company, London, Amsterdam, Ontario, Sydney, Tokyo, 1983. 